\title{
The prevention and management of rabies
}

This Clinical Review (BMJ 2015;350:g7827, doi:10.1136/bmj. g7827) contains an error in the first paragraph of the section on "Routes for administering vaccines." The third sentence should have read: "The intradermal route has the advantages of being dose sparing, resulting in equivalent protection at up to $60-80 \%$ of the cost of the intramuscular route, and requiring a single visit for post-exposure prophylaxis in people with documented previous complete pre-exposure or post-exposure prophylaxis."

Cite this as: BMJ 2015;350:h575

๑ BMJ Publishing Group Ltd 2015 